\title{
X-ray microanalysis of electro-erosive powder materials, obtained from tungsten-nickel-iron (TNI) alloy wastes in water
}

\author{
E.V. Ageev ${ }^{1 *}$, V.L. Selyutin ${ }^{1}$, and S.V. Pikalov ${ }^{1}$ \\ ${ }^{1}$ Southwest State University, 305040 Kursk, Russian Federation
}

\begin{abstract}
This article presents the results of elemental composition study of the electro-erosion powders, obtained from waste alloy TNI in water. It is established that the main elements are tungsten, nickel, iron and oxygen.
\end{abstract}

\section{Introduction}

Heavy alloys have a number of very valuable properties, due to which they are effectively used in many industries. Currently, one of the main problems of using these alloys is the presence in it of a significant amount of expensive tungsten [1-12]. This problem can be solved by grinding their waste and reuse. Existing industrial grinding technologies are distinguished by large tonnage, high energy costs and environmental problems. One of the promising and industrially inapplicable methods of grinding any electrically conductive material is the electroerosion method [13-19].

To develop technologies for the reuse of electro-erosion powders obtained from TNI alloy, and to evaluate the effectiveness of their use, complex theoretical and experimental studies are required.

The aim of the work was to study the elemental composition of electroerosive powders, obtained from TNI alloy waste in wate.

\section{Materials and Methods}

Powders were obtained at the installation of electroerosive dispersion protected by RF Patent No. 2449859, from TNI alloy shavings. Electronic probe microanalysis (EPM) was carried out with the use of EDAX firm manufacture energy-dispersive x-ray emission analyzer installed into Nova NanoSEM 450 scanning-electron microscope. Using the method of scanning electron microscopy by means of a secondary electrons detector powders sample particles were analyzed.

With the use of EDAX firm manufacture energy-dispersive $\mathrm{x}$-ray emission analyzer installed into Nova NanoSEM 450 scanning-electron microscope characteristic X-ray radiation spectra in different points on the surface of powders sample were obtained.

* Corresponding author: ageev_ev@mail.ru 
Determination of the elemental composition of microscopic objects from characteristic $\mathrm{X}$-ray radiation being excited in them is meant by electron probe microanalysis. Spectrometers of two types (crystal-free spectrometer or analyzing crystal-type spectrometer) are used in electron probe microanalysis for characteristic spectrum analysis (CSA). The electron-optical system of a scanning-electron microscope is the base for CSA. During the interaction of the electron probe with the sample (Fig. 1 and 1) X-ray radiation is one of the signals being excited. The X-ray radiation can be divided in characteristic and deceleration radiation.

Deceleration X-ray radiation occurs in consequence of primary electrons deceleration in the electrical (Coulomb) field of the atoms of material being analyzed. In this case the kinetic energy of primary electrons is partially or completely transformed into X-ray radiation energy. Consequently, the radiation has a continuous spectrum with the energy from zero level to the energy of a bombarding electron. That is why the radiation is also called continuous X-ray radiation. When carrying out electron probe microanalysis deceleration radiation is undesirable, because it makes the main contribution to increasing background radiation intensity and cannot be excluded.

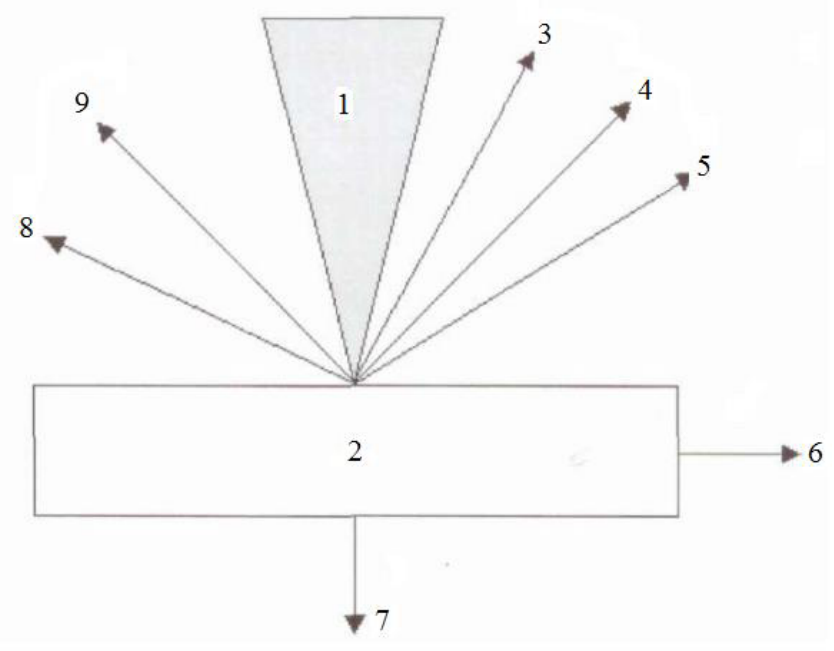

Fig. 1. Effects of the interaction of an electron ray with the object: 1 - electron ray, 2 - object, 3 reflected electrons, 4 - secondary electrons, 5 - Auger electrons, 6 - absorbed electrons current 7 passed electrons, 8 - cathodoluminescent radiation, 9 -X-ray radiation.

When primary electrons penetrate a sample, they are decelerated not only by the electrical field of atoms, but also in consequence of immediate collision with the electrons of the material atoms. As a result primary electrons can knock out electrons from the internal K-, L-, or M-shells, leaving an atom of the sample in excited state. Vacancy sites are filled through passage of electrons from higher energy levels. The atom is passed to the main state, and excess energy is released in the form of an X-ray quantum. As far as the energy of the quantum formed depends only on the energy of electron levels involved in the process, and such electron levels are typical for every element, characteristic X-ray radiation occurs. As every atom has a well-defined finite number of levels, between which not only certain type passages are possible, characteristic X-ray radiation forms a discrete linear spectrum. 


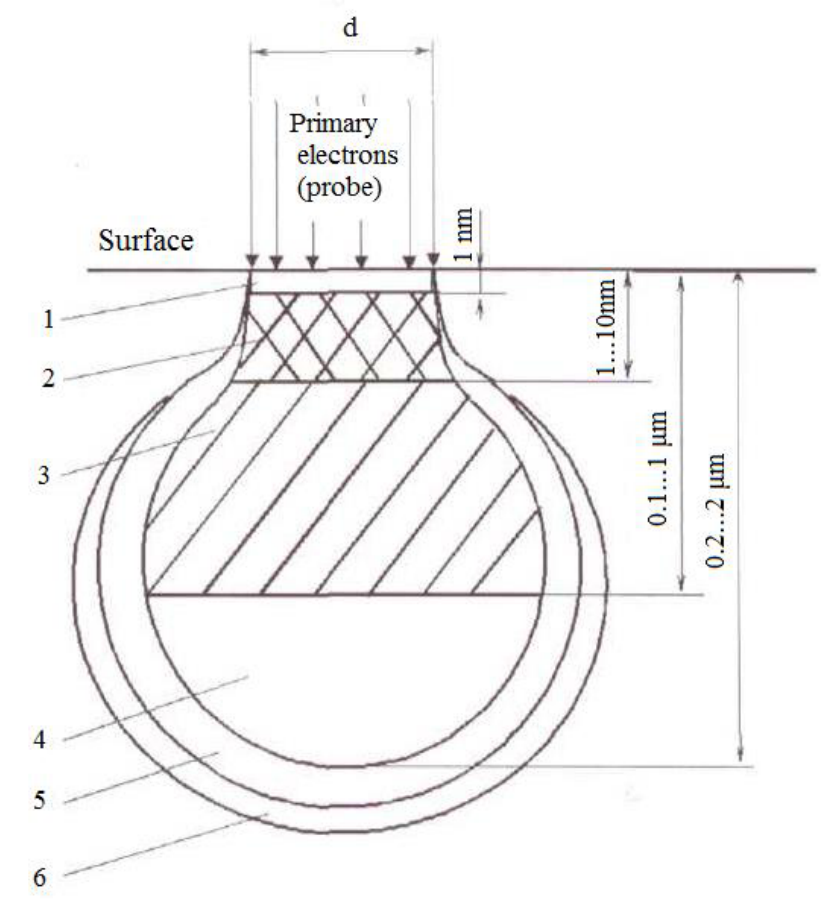

Fig.2. . Signal areas and space resolution under irradiation of the object surface with a flow of electrons (probe). Areas of generation of: 1 - auger electrons, 2 - secondary electrons, 3 -reflected electrons, 4 - characteristic X-ray radiation, 5 - deceleration X-ray radiation, 6 - fluorescence.

It does not succeed to determine light elements with an atomic number less than 4 within an alloy by means of electron probe microanalysis. The same difficulties with elements determination occur, when the lines of L- or M-series of one element are overlaid by the lines of K-series of another element. An important characteristic of CSA is its locality, i. e. the volume of material, in which characteristic X-ray radiation is excited. The volume is determined primarily by the diameter of an electron probe on a sample and depends on accelerating voltage and elemental composition of the material (Figure 1).

Elements distribution analysis can be carried out in qualitative, semi-quantitative, and quantitative form. Qualitative analysis allows determining the type of elements composing an area of a sample being analyzed. If a sample has several phases (regions), the elemental composition of which in unknown, qualitative analysis of each phase is to be carried out. Usually qualitative analysis is used for determining the character of elements distribution along the area of a polished section. After qualitative analysis quantitative analysis is often carried out in separate points chosen. According to the information the obtained software allows determining a phase type on the basis of its elemental composition. Semiquantitative analysis is carried out, if it is necessary to determine elements distribution along the lines (linear analysis). Linear analysis is carried out by the method of step scanning, i.e. by consequent carrying out analysis in certain points. Thus, quantitative determination of elements concentration is carried out with a specified degree of accuracy.

\section{Results}

To refine the profile of experimental X-ray diffraction patterns, the PDXL RIGAKU software package was used. The background was subtracted by the Sonneveld - Wisser 
method, the experimental profile was smoothed by the Savitsky - Golaya method, and the components $\mathrm{k}_{\alpha 1}$ and $\mathrm{k}_{\alpha 2}$ were separated by the Rachinger method. To describe the diffraction maxima, a superposition of the Gauss function and the Lorentz function was used. The phase composition was determined using the ICCD PDF-2 database (2014).

The results of X-ray microanalysis are shown in Figure 3.

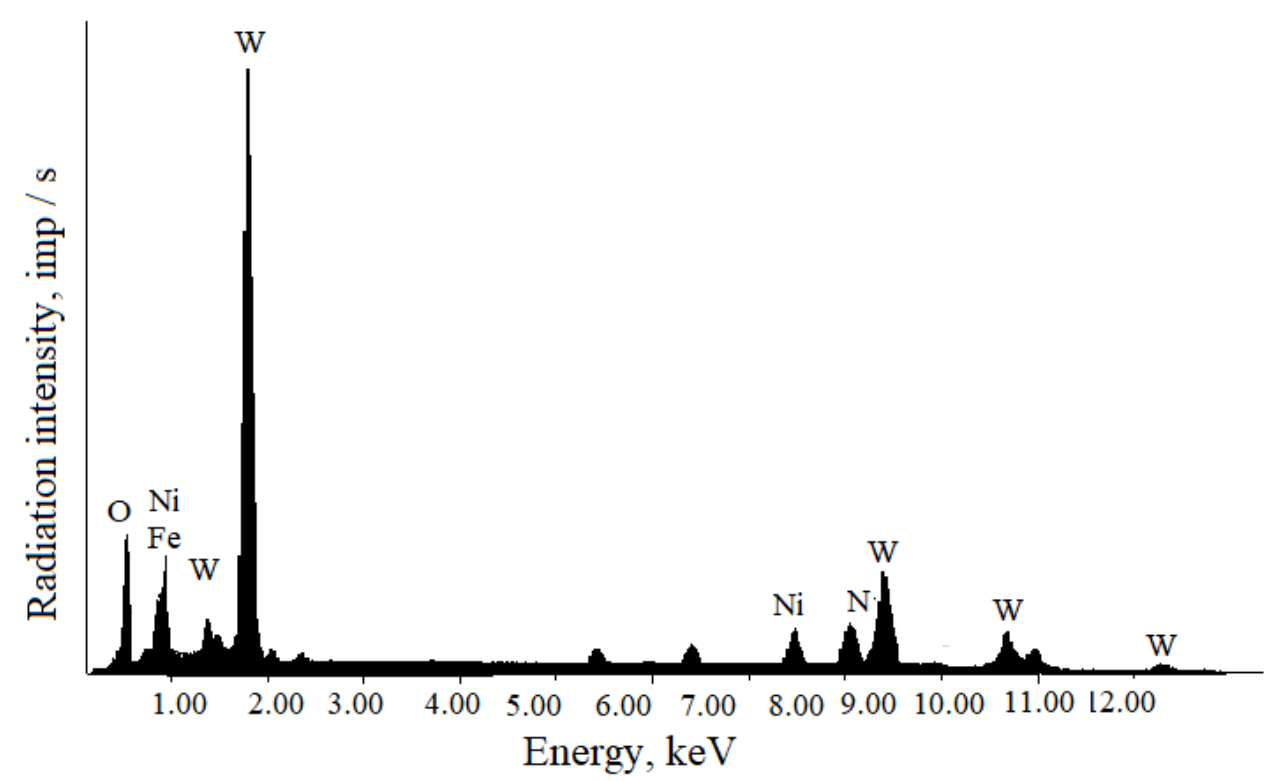

Fig.3. X-ray microanalysis on the surface of electro-erosion TNI alloy particles in distilled water.

\section{Conclusion}

Based on the elemental composition study of the electro-erosion powders, obtained from waste of a TNI alloy in water, it was found that the main elements are tungsten, nickel, iron and oxygen. The study will determine the most relevant application area of the obtained samples and improve the quality of scientific and technological developments.

This work was supported by a grant from the President of the Russian Federation (NSh-2564.2020.8).

\section{References}

1. M.I. Janitor, Development of physico-chemical and technological fundamentals of the processing of tungsten-cobalt hard alloy by electroerosive dispersion: diss. ... cand. tech. Sciences / Dvornik Maxim Ivanovich, 116 (Khabarovsk, 2006)

2. M. Sarwar, M. Persson, H. Hellbergh, Wear, 259, 1144-1150 (2005)

3. A. Basu, J. Chakraborty, S.M. Shariff, G. Padmanabham, S.V. Joshi, G. Sundararajan, J. Dutta Majumdar, I. Manna, Scr. Mat., 56, 887-890 (2007)

4. V.A. Pozdnyakov, V.T. Borisov, V.T. Molotilov, Russ. Met., 6, 583-586 (2002)

5. J. Epp, H. Surm, O. Kessler, T. Hirsch, Act. Mat., 55, 5959-5967 (2007)

6. E.A. Deulin, R.A. Nevshupa, Appl. Surf. Sc., 144-145, 283-286 (1999)

7. H. Överstam, J. of Mat. Proc. Tech., 171, 446-450 (2006)

8. J. Zhang, Z. Zhang, W. Liu, Q. Xue, Wear, 219, 184-187 (1998)

9. S. Jiang, H. Mao, J. of Trib., 133, 2101 (2011) 
10. P. Harlin, P. Carlsson, U. Bexell, M. Olsson, Surf. and Coat. Tech., 201, 4253-4259 (2006)

11. B. Podgornik, J. Vižintin, Mat. Scien. and Engin., 315, 28-34 (2001)

12. B.R. Lazarenko, N.I. Lazarenko, A. p. 70000 USSR, B 22f, 09/00 A method for producing powders and a device for its implementation. - No. 1371/321510; declared 04/01/1943; publ. 09/23/1964, Bull. 22, 2.

13. V.N. Gusev, Anodic-mechanical processing of metals, 321 (1952)

14. E.V. Ageev, Hard.techn.and coat., 6, 8-14 (2011)

15. R.A. Latypov, G.R. Latypova, E.V. Ageev, P.I. Burak, ISJ, 5, 80-86 (2013)

16. E.V. Ageev, Electromet., 10, 24-27 (2011)

17. A. Pereverzev, E. Ageev, MATEC Web of Conf., 298, 00037 (2019)

18. E.V. Ageev, S.V. Khardikov, E.A. Vorobyev, A.A. Sysoev, MATEC Web of Conf., 298, 00127 (2019)

19. R.A. Latypov, E.V. Ageeva, G.R. Latypova, MATEC Web of Conf., 298, 00125 (2019) 